\title{
Finite Element Formulation of the General Magnetostatic Problem in the Space of Solenoidal Vector Functions
}

\author{
By Mark J. Friedman
}

\begin{abstract}
A new finite element method for the solution of the general magnetostatic problem is formulated and analyzed. The space of trial functions consists of solenoidal piecewise polynomial vector functions. We start with an integral formulation, in terms of the flux density, in the domain occupied by magnetic material. Using the properties [10] of the spectrum of the relevant singular integral operator we derive a weak formulation involving an integral operator on the boundary only. Thus the resulting finite element matrix consists of a sparse part corresponding to the interior of the iron domain and a full part corresponding to the boundary. Using the method of monotone operators, existence and uniqueness of the solution of the weak formulation as well as its discretization are proven. Error estimates are derived with the special emphasis on the case when magnetic permeability is large. Finally, solution of the problem by successive iteration is analyzed.
\end{abstract}

\section{Introduction.}

1.1. In this paper we analyze the general (i.e., nonlinear, nonhomogeneous and anisotropic) magnetostatic problem without hysteresis, and develop a new finite element method for its numerical solution. The problem is to calculate the magnetic field inside and outside of a ferromagnetic material placed in a given field (produced by currents in free space). One encounters the problems of this type in, for example, the design of electromagnets, electrical motors and other electromagnetic devices.

Currently, the magnetostatic problems have been solved by using a differential interface formulation in the whole space or an integral formulation in a bounded ferromagnetic domain. The numerical methods based on the differential formulations have been considered, e.g., in [4], [11], [16], [24], [25]. For the numerical methods based on integral formulations, see, e.g., [1], [2], [5]-[7], [20], [22] and references therein. Recently some "coupled" finite element-boundary solution procedures have been introduced (see, e.g., [3], [15], [17], [8], [9], [21] and the discussion there). The common features of these coupled methods are that they lead to sparse matrix systems in the interior of the ferromagnetic domain and full matrix systems only on the boundary nodes.

As far as we are aware, the first detailed mathematical analysis of the magnetostatic problem has been done by I. Mayergoyz [16] for the differential formulation and by the author in [5], [7] and the present paper for the integral formulation.

Received October 13, 1982; revised March 17, 1983 and October 24, 1983.

1980 Mathematics Subject Classification. Primary 45C05, 45L10, 65J15, 65R20, 78A30. 
Similar results have been obtained by J. E. Pasciak [21]. The later paper also contains a numerical procedure (similar to the one suggested by the author and J. S. Colonias in [8] and by the author in [9]) and the results of numerical experiments.

1.2. Let us now describe the problem more precisely. Let $\Omega \subset \subset R^{3}$ be a bounded domain, containing the ferromagnetic material, with Lipschitz boundary $\Gamma$. We will denote by $\bar{\Omega}$ the closure of $\Omega$, and the notation $\mathbf{u}(x)=\left(u_{1}(x), u_{2}(x), u_{3}(x)\right)$ will stand for a vector function on $R^{3}$. We also set $u \equiv|\mathbf{u}|=\sqrt{u_{1}^{2}+u_{2}^{2}+u_{3}^{2}}$.

Maxwell's equations for magnetostatics are given in terms of the flux density $\mathbf{B}$, the net field $\mathbf{H}$ and the current density $\delta$ :

$$
\nabla \cdot \mathbf{B}=0, \quad \nabla \times \mathbf{H}=\boldsymbol{\delta} .
$$

These equations are connected by a constitutive relation $\mathbf{H}(x, \mathbf{B})$ giving $\mathbf{H}$ as a function of $x$ and $\mathbf{B}$; this relation is determined experimentally. We shall also often use an operator notation

$$
(h \mathbf{B})(x) \equiv \mathbf{H}(x, \mathbf{B}): \Omega \times R^{3} \rightarrow R^{3} .
$$

Throughout the exterior region $\Omega^{\prime}=R^{3} / \bar{\Omega}$ we always have $\mathbf{H}=\mathbf{B}$. In the isotropic case, (1.1) is usually written using the permeability $\mu$, as

$$
\mathbf{H}(x, \mathbf{B})=\frac{\mathbf{B}(x)}{\mu(x, B)} .
$$

In addition, the normal component of the $\mathbf{B}$ field and the tangential components of the $\mathbf{H}$ field are continuous across $\Gamma$, and

$$
B=O\left(|x|^{-2}\right) \text { as } x \rightarrow \infty .
$$

One way of reformulating the above problem is by the introduction of the volume integral operator, call it $A$, defined by

$$
(A \mathbf{M})(x) \equiv \frac{1}{4 \pi} \nabla \int_{\Omega} \mathbf{M}(y) \cdot \nabla_{y} \frac{1}{r} d y, \quad r=|x-y|,
$$

where $A$ maps vector functions defined on $\Omega$ into vector functions on $R^{3}$.

Let $\mathbf{B}_{a}$ be a given "applied field", i.e., the field due to currents in free space:

$$
\mathbf{B}_{a}(x)=\frac{1}{4 \pi} \int \delta(y) \times \nabla_{y} \frac{1}{r} d y .
$$

Note that $\mathbf{B}_{a}$ can be calculated by analytical or numerical integration from the known current density $\boldsymbol{\delta}$.

Define the magnetization $\mathbf{M}$ by

$$
\mathbf{M}=\mathbf{B}-\mathbf{H} \equiv \mathbf{B}-h \mathbf{B} .
$$

Now $-A \mathbf{M}$ in (1.3) represents the demagnetization field due to spatial distribution of magnetization. Since, as is well known, the net field is the sum of the appplied field and the demagnetization field, by combining the above identities one can easily derive the nonlinear singular integral equation

$$
R \mathbf{B} \equiv h \mathbf{B}+A(\mathbf{B}-h \mathbf{B})=\mathbf{B}_{a} .
$$

After (1.6) is solved the field outside the ferromagnetic material, in $\Omega^{\prime}$, is given by

$$
\mathbf{B}(x)=\mathbf{B}_{a}(x)-(A(\mathbf{B}-h \mathbf{B}))(x), \quad x \in \Omega^{\prime} .
$$


This can also be written as

$$
\mathbf{B}(x)=\mathbf{B}_{a}(x)-\frac{1}{4 \pi} \nabla \int_{\Gamma} \frac{\sigma(y)}{r} d \gamma_{y},
$$

where $\boldsymbol{\sigma}$ is (almost) $\mathbf{M} \cdot \mathbf{n}$, the normal component of magnetization on $\Gamma$; see Section 3 for details. In practice, the outside field is often of most interest.

We now briefly describe our method for solving (1.6). (Details will be given in Section 3). As a result of Maxwell's equations $\nabla \cdot \mathbf{B}=0, \mathbf{B}_{a}$ and $\mathbf{B}$ are both solenoidal. Let $J$ denote a space of solenoidal (divergence-free) vector functions. Letting $J_{h} \subseteq J$ be a family of finite-dimensional spaces, an obvious projection method would be to find $\mathbf{B}_{h} \in J_{h}$ such that

$$
\int_{\Omega} R \mathbf{B}_{h} \cdot \mathbf{u} d x=\int_{\Omega} \mathbf{B}_{a} \cdot \mathbf{u} d x \text { for all } u \in J_{h} .
$$

Even if $J_{h}$ has a local basis, this formulation leads to a full matrix over the volume $\Omega$. We define $\mathbf{B}_{h} \in J_{h}$ by

$$
\begin{aligned}
& \int_{\Omega} h \mathbf{B}_{h} \cdot \mathbf{u} d x+\frac{1}{4 \pi} \int_{\Gamma} \int_{\Gamma} \frac{1}{r}\left(\mathbf{B}_{h}-P_{J}^{h} h \mathbf{B}_{h}\right)(y) \cdot \mathbf{n}_{y} d \gamma_{y} \mathbf{u}(x) \cdot \mathbf{n}_{x} d \gamma_{x} \\
&=\int_{\Omega} \mathbf{B}_{a} \cdot \mathbf{u} d x \quad \text { for all } \mathbf{u} \in J_{h},
\end{aligned}
$$

where $P_{J}^{h}$ is the orthogonal projector onto $J_{h}$. From (1.10), defining $\sigma_{h}$ from $\mathbf{B}_{h}$, by (1.8) it follows that the error in the field outside the magnetic material is determined by the accuracy in $\sigma-\sigma_{h}$ on $\Gamma$, more precisely,

$$
\begin{aligned}
\left|\mathbf{B}(x)-\mathbf{B}_{h}(x)\right| & =\frac{1}{4 \pi}\left|\int_{\Gamma}\left[\sigma(y)-\sigma_{h}(y)\right] \nabla_{y} \frac{1}{r} d \gamma_{y}\right| \\
& \leqslant \frac{1}{4 \pi}\left\|\nabla \frac{1}{r}\right\|_{1 / 2, \Gamma}\left\|\boldsymbol{\sigma}-\sigma_{h}\right\|_{-1 / 2, \Gamma},
\end{aligned}
$$

where $\|\cdot\|_{s, \Gamma}$ stands for the norm in the Sobolev space $H^{s}(\Gamma)$ (cf. [18]). In Section 4, we shall derive estimates ((4.15) and (4.16)) for $\sigma-\sigma_{h}$, which together, show that we have good approximation over the whole range of permeability, even in the limit $\mu \rightarrow \infty$.

Compared to (1.9) and the usual volume integral method (based on straightforward discretization of (1.6)) this has the advantage that, given a local basis for $J_{h}$, the system arising from the volume integral is sparse. Thus, a dense matrix enters only from the boundary terms. In addition to the point just mentioned, it turns out that, for (1.9), the errors become large with high permeability (see, e.g., [20] and the discussion there). The reason for this is, roughly speaking, that the lower bound of the spectrum of the corresponding (positive definite) operator approaches zero. This leads to a large constant in error estimates and also increases considerably the condition number of the finite-dimensional problem so that an iterative method of solution would be less efficient.

1.3. The outline of the remainder of the paper is as follows. In Section 2, we introduce notation and give some preliminary results. We decompose $[10]\left(L^{2}(\Omega)\right)^{3}$ into invariant subspaces where the operator $A$ is the zero map, the identity map and a subspace where $A$ is positive definite and bounded (extending the results of [5], [7] to the case when $\Omega$ is not simply connected and $\Gamma$ is Lipschitz). 
In Section 3, we first present the formulation (3.1) of our problem in the space $J \subset \mathbf{L}^{2}$ of generalized solenoidal ("divergence-free" in a weak sense) vector functions. Then we define our finite element method for the numerical solution of (3.1) and, using the spectral decomposition of $A$, prove some auxiliary results required for the error analysis in Section 4.

In Section 4, we prove existence and uniqueness of the solution, using the monotone operator method, and derive error estimates.

In Section 5, we analyze the successive iteration method for the iterative solution of our problem.

In Theorem 5.2, we derive an estimate which shows that the convergence does not deteriorate over the whole range of permeability, provided an appropriate choice of the initial approximation is made.

2. Notation annd Preliminaries. We shall denote by $H^{s}(\Omega)$ and $H^{s}(\Gamma)$ Sobolev spaces on $\Omega$ and $\Gamma$ of order $s$ with corresponding norms $\|\cdot\|_{s, \Omega}$ and $\|\cdot\|_{s, \Gamma}$, respectively [13], [18]. For negative $s$, the Sobolev spaces are defined by duality. Let $\mathscr{D}(\Omega)$ (or $\mathscr{D}(\bar{\Omega})$ ) be the space of $\mathscr{C}^{\infty}$ functions with compact support contained in $\Omega$ (or in $\bar{\Omega}$ ). The closure of $\mathscr{D}(\Omega)$ in $H^{s}(\Omega)$ is denoted by $H_{0}^{s}(\Omega) . \mathscr{D}(\bar{\Omega})$ is dense in $H^{s}(\Omega)$. The notation $\mathbf{H}$ will stand for the product space $H^{3}$ which has components in the space $H$. We shall also write $\mathbf{L}^{2}$ for $\mathbf{L}^{2}(\Omega)$. When $H$ is a Hilbert space $\mathbf{H}$ inherits the obvious norms and inner products. Let $(\cdot, \cdot)$ denote the $L^{2}$ inner product on $\Omega$ given by

$$
(u, v)=\int_{\Omega} u v d x
$$

The corresponding $\mathbf{L}^{2}$ inner product is

$$
(\mathbf{u}, \mathbf{v})=\int_{\Omega} \mathbf{u} \cdot \mathbf{v} d x
$$

We shall need some auxiliary subspaces of $\mathbf{L}^{2}$. Define

$$
\begin{aligned}
G & =\left\{\mathbf{u}: \mathbf{u}=\nabla \phi, \phi \in H^{1}(\Omega)\right\} . \\
\stackrel{\circ}{G} & =\left\{\mathbf{u}: \mathbf{u}=\nabla \phi, \phi \in H_{0}^{1}(\Omega)\right\}=\text { closure in } \mathbf{L}^{2} \text { of } \dot{G} \\
& =\{\mathbf{u}: \mathbf{u}=\nabla \phi, \phi \in \mathscr{D}(\Omega)\} . \\
U & =\text { the orthogonal complement of } \stackrel{\circ}{ } \text { in } G . \\
\dot{J} & =\text { the orthogonal complement of } G \text { in } \mathbf{L}^{2} . \\
J & =\left\{\mathbf{u} \in \mathbf{L}^{2}: \nabla \cdot \mathbf{u}=0\right\} .
\end{aligned}
$$

The following results for the operator $A$ are proven in [10].

THEOREM 2.1. The operator $A$ is a bounded selfadjoint map on $\mathbf{L}^{2}$ and satisfies

(i) $\operatorname{Ker} A=\dot{J}$.

(ii) $A$ is the identity when restricted to $\dot{G}^{\circ}$.

(iii) $U$ is an invariant subspace of $A$.

(iv) The spectrum of $A$ on $U$ is contained in the interval $\left[\lambda_{0}, \Lambda_{0}\right]$ where $0<\lambda_{0} \leqslant \Lambda_{0}$ $\leqslant 1$ are positive constants which depend only on $\Omega$. 
We next give an additional characterization of the spaces $U, \dot{J}$ and $J$. Denote

$$
E=\left\{\mathbf{u} \in \mathbf{L}^{2}: \nabla \cdot \mathbf{u} \in \mathbf{L}^{2}\right\} .
$$

Lemma 2.1 [26, TheOREM 1.2, Remark 1.3]. There exists a unique linear continuous operator $\gamma_{n}: E \rightarrow H^{-1 / 2}(\Gamma)$ such that

$$
\gamma_{n} \mathbf{u}=\text { the restriction of } \mathbf{u} \cdot \mathbf{n} \text { to } \Gamma \text { for all } \mathbf{u} \in \mathscr{D}(\bar{\Omega}),
$$

where $\mathbf{n}$ is the outward normal (which exists almost everywhere on $\Gamma[17$, Lemma 2.4.2]).

In what follows we shall use the notation

$$
\left.\mathbf{u} \cdot \mathbf{n}\right|_{\Gamma}=\gamma_{n} \mathbf{u} \quad \forall \mathbf{u} \in E .
$$

LEMMA 2.2 [26, THEOREM 1.4].

$$
\begin{aligned}
\stackrel{\circ}{J} & =\left\{\mathbf{u} \in E: \nabla \cdot \mathbf{u}=0,\left.\mathbf{u} \cdot \mathbf{n}\right|_{\Gamma}=0\right\}=\text { closure in } \mathbf{L}^{2} \text { of } \dot{J} \\
& =\{\mathbf{u} \in \mathscr{D}(\Omega): \nabla \cdot \mathbf{u}=0\} .
\end{aligned}
$$

LEMMA 2.3 .

$$
\begin{aligned}
J & =\text { closure in } \mathbf{L}^{2} \text { of } \tilde{J}=\{\mathbf{u} \in \mathscr{D}(\overline{\mathbf{\Omega}}): \nabla \cdot \mathbf{u}=0\} . \\
\mathbf{L}^{2} & =\stackrel{\circ}{G} \oplus J . \\
J & =U \oplus \dot{J} . \\
U & =\left\{\mathbf{u}: \mathbf{u}=\nabla \phi, \phi \in H^{1}(\Omega), \Delta \phi=0\right\} .
\end{aligned}
$$

Proof. Let $\mathbf{u} \in J$. From the definition of $J, J \subset E$. By [25, Theorem 1.1] $\mathscr{D}(\bar{\Omega})$ is dense in $E$. Let $\left\{\mathbf{u}_{n}\right\}_{n=1}^{\infty} \subset \mathscr{D}(\bar{\Omega})$ be a sequence converging to $\mathbf{u}$ in $E$ as $n \rightarrow \infty$, i.e.,

$$
\left\|\mathbf{u}_{n}-\mathbf{u}\right\| \rightarrow 0 \text { as } n \rightarrow \infty, \quad\left\|\nabla \cdot \mathbf{u}_{n}\right\| \rightarrow 0 \quad \text { as } n \rightarrow \infty .
$$

Then

$$
\tilde{\mathbf{u}}_{n}=\mathbf{u}_{n}-\nabla \frac{1}{4 \pi} \int_{\Omega} \frac{\nabla \cdot \mathbf{u}_{n}(y)}{r} d y \in \tilde{J}
$$

and converges to $\mathbf{u}$ in $E$ as $n \rightarrow \infty$. Since $J$ is clearly a Hilbert space, with the $L^{2}$-norm, we have (2.4).

Let now $\mathbf{u} \in \mathbf{L}^{2}$ be orthogonal to $\stackrel{G}{G}$. Then

$$
(\mathbf{u}, \nabla \phi)=0 \text { for all } \phi \in \mathscr{D}(\Omega),
$$

which means that $\nabla \cdot \mathbf{u}=0$ in the sense of distributions, which gives (2.5). Together with the definitions of $U$ and $\dot{J}$, (2.5) implies (2.6). Since $U \subset G$ for any $\mathbf{u} \in U$ we have $\mathbf{u}=\nabla \phi, \phi \in H^{1}(\Omega)$. From (2.6) and the definition of $J$ we also have that $\Delta \phi=0$ in $\Omega$, which gives (2.7).

Throughout this paper, $C$ will denote a generic constant, not necessarily the same at different occurrences.

3. Formulation of the Problem. The Numerical Procedure. From Maxwell's equations for magnetostatics $\nabla \cdot \mathbf{B}=0$. Therefore, $J$ turns out to be a natural space for the solution of (1.6).

The variational formulation of (1.6) in $J$ is for a given $\mathbf{B}_{a} \in J$ to find $\mathbf{B} \in J$ so that

$$
(R \mathbf{B}, \mathbf{u})=\left(\mathbf{B}_{a}, \mathbf{u}\right) \quad \forall \mathbf{u} \in J
$$


Let $J_{h} \subset J$ be a family of finite-dimensional subspaces of $J$. We define the approximate solution $\mathbf{B}_{h} \in J_{h}$ of the problem (3.1) by

$$
\begin{aligned}
\left(R_{h} \mathbf{B}_{h}, \mathbf{u}\right) \equiv & \int_{\Omega} \mathbf{H}\left(x, \mathbf{B}_{h}(x)\right) \cdot \mathbf{u}(x) d x \\
& +\int_{\Gamma} \int_{\Gamma}(1 / r)\left(\mathbf{B}_{h}(y)-\left(P_{J}^{h} h \mathbf{B}_{h}\right)(y)\right) \cdot \mathbf{n}_{y} d \gamma_{y} \mathbf{u}(x) \cdot \mathbf{n}_{x} d \gamma_{x} \\
= & \int_{\Omega} \mathbf{B}_{a}(x) \cdot \mathbf{u}(x) d x \quad \forall \mathbf{u} \in J_{h} .
\end{aligned}
$$

We shall establish below that this formulation is indeed a discretization of (3.1). The following simple lemma is crucial for our numerical method. Denote by $P_{v}$ the orthogonal projector of $\mathbf{L}^{2}$ onto $V \subset \mathbf{L}^{2}$.

LEMMA 3.1.

$$
\begin{aligned}
(A \mathbf{M}, \mathbf{u}) & =\left(A P_{J} \mathbf{M}, \mathbf{u}\right) \quad \forall \mathbf{M} \in \mathbf{L}^{2}, \forall \mathbf{u} \in J, \\
\forall \mathbf{u} & \in J,\left.\quad\left(P_{U} \mathbf{u}\right) \cdot \mathbf{n}\right|_{\Gamma}=\left.\mathbf{u} \cdot \mathbf{n}\right|_{\Gamma} \in H^{-1 / 2}(\Gamma) .
\end{aligned}
$$

Proof. By (2.5) and (ii) of Theorem 2.1 we have (3.3):

(3.5) $(A \mathbf{M}, \mathbf{u})=\left(A \mathbf{M}, P_{J} \mathbf{u}\right)=\left(P_{J} A \mathbf{M}, \mathbf{u}\right)=\left(P_{J}\left(A P_{J} \mathbf{M}+P_{\dot{G}} \mathbf{M}\right), \mathbf{u}\right)=\left(A P_{J} \mathbf{M}, \mathbf{u}\right)$.

From (2.6) for $\mathbf{u} \in J, \mathbf{u}=P_{U} \mathbf{u}+P_{j} \mathbf{u}$. And (3.4) follows from the fact that by (2.3) $\left.\left(P_{j} \mathbf{u}\right) \cdot \mathbf{n}\right|_{\Gamma}=0$.

THEOREM 3.1. (i) The formulation

$$
(R \mathbf{B}, \mathbf{u}) \equiv(h \mathbf{B}, \mathbf{u})+\left(A\left(\mathbf{B}-P_{J} h \mathbf{B}\right), \mathbf{u}\right)=\left(\mathbf{B}_{a}, \mathbf{u}\right) \quad \forall \mathbf{u} \in J
$$

is equivalent to (3.1).

(ii) The formulation (3.2) makes sense in $J_{h}$, and it is equivalent to

$$
\left(R_{h} \mathbf{B}_{h}, \mathbf{u}\right) \equiv\left(h \mathbf{B}_{h}, \mathbf{u}\right)+\left(A\left(\mathbf{B}_{h}-P_{J}^{h} h \mathbf{B}_{h}\right), \mathbf{u}\right)=\left(\mathbf{B}_{a}, \mathbf{u}\right) \quad \forall \mathbf{u} \in J_{h},
$$

where $P_{J}^{h} \equiv P_{J^{h}}$.

Remark 3.1. The formulations (3.6) and (3.7) are used for the analysis of the problem and the derivation of the error estimates, while the formulation (3.2) is used for the numerical solution.

Proof. Statement (i) is an immediate consequence of (3.3). For $\mathbf{u} \in J$, integrating by parts in (1.3),

$$
\begin{aligned}
4 \pi(A \mathbf{u})(x) & =\nabla\left[\int_{\Gamma}(1 / r) \mathbf{u}(y) \cdot \mathbf{n}_{y} d \gamma_{y}-\int_{\Gamma}(1 / r) \nabla \cdot \mathbf{u}(y) d y\right] \\
& =\nabla \int_{\Gamma}(1 / r) \mathbf{u}(y) \cdot \mathbf{n}_{y} d \gamma_{y} \quad \forall x \in \Omega .
\end{aligned}
$$

Therefore for any $\mathbf{u}, \mathbf{v} \in J$ we have, integrating by parts,

$$
\begin{aligned}
4 \pi(A \mathbf{u}, \mathbf{v}) & =\int_{\Omega} \nabla \int_{\Gamma}(1 / r) \mathbf{u}(y) \cdot \mathbf{n}_{y} d \gamma_{y} \cdot \mathbf{v}(x) d x \\
& =\int_{\Gamma} \int_{\Gamma}(1 / r) \mathbf{u}(y) \cdot \mathbf{n}_{y} d \gamma_{y} \mathbf{v}(x) \cdot \mathbf{n}_{x} d \gamma_{x} .
\end{aligned}
$$


Now from Theorem 2.1, (iv), Lemma 2.1 and (2.4) we have

$$
(A \mathbf{u}, \mathbf{v}) \leqslant \Lambda_{0}\|\mathbf{u}\|\|\mathbf{v}\| \leqslant \Lambda_{0} C\|\mathbf{u} \cdot \mathbf{n}\|_{-1 / 2, \Gamma}\|\mathbf{v} \cdot \mathbf{n}\|_{-1 / 2, \Gamma} \quad \forall \mathbf{u}, \mathbf{v} \in J .
$$

Therefore the right-hand side of (3.8) is a bounded bilinear form in $H^{-1 / 2}(\Gamma) \times$ $H^{-1 / 2}(\Gamma)$. Thus (3.8) holds by (2.4).

After (3.2) is solved, the approximate field outside the magnetic material, similarly to $(1.7)$, is given by

$$
\mathbf{B}_{h}(x)=\mathbf{B}_{a}(x)-\frac{1}{4 \pi} \nabla \int_{\Omega} \mathbf{M}_{h}(y) \cdot \nabla_{y}(1 / r) d y, \quad x \in \Omega^{\prime},
$$

where

$$
\mathbf{M}_{h}=\mathbf{B}_{h}-\mathbf{H}_{h} \equiv \mathbf{B}_{h}-h \mathbf{B}_{h}
$$

Denote

$$
\begin{gathered}
\boldsymbol{\sigma}=\left.\left(P_{U} \mathbf{M}\right) \cdot \mathbf{n}\right|_{\Gamma}, \\
\boldsymbol{\sigma}_{h}=\left.\left(P_{J}^{h} \mathbf{M}_{h}\right) \cdot \mathbf{n}\right|_{\Gamma} .
\end{gathered}
$$

THEOREM 3.2.

$$
\sigma_{h}=\left.\left(P_{U} P_{J}^{h} \mathbf{M}_{h}\right) \cdot \mathbf{n}\right|_{\Gamma} .
$$

The exact field (1.7) and the approximate one (3.10), outside magnetic material, can be written, respectively, as

$$
\begin{array}{cc}
\mathbf{B}(x)=\mathbf{B}_{a}(x)-\frac{1}{4 \pi} \nabla \int_{\Gamma}(1 / r) \sigma(y) d \gamma_{y}, & x \in \Omega^{\prime}, \\
\mathbf{B}_{h}(x)=\mathbf{B}_{a}(x)-\frac{1}{4 \pi} \nabla \int_{\Gamma}(1 / r) \sigma_{h}(y) d \gamma_{y}, & x \in \Omega^{\prime} .
\end{array}
$$

Proof. Equation (3.14) follows from (3.4) with $\mathbf{u}=\left(P_{J}^{h} \mathbf{M}_{h}\right)$. From the argument similar to the one in the proof of Theorem 3.1, (ii), using (3.4) it follows that for $\mathbf{u} \in J$

$$
(A \mathbf{u})(x)=\frac{1}{4 \pi} \nabla \int_{\Gamma}(1 / r)\left(P_{U} \mathbf{u}\right)(y) \cdot \mathbf{n}_{y} d \gamma_{y} .
$$

Now by (2.5) $\mathbf{L}^{2}=J \oplus \stackrel{\circ}{\text {, }}$, and therefore to verify (3.15) and (3.16) we need only to show that for any $\mathbf{u} \in \stackrel{\circ}{\mathrm{G}}$

$$
\nabla \int_{\Omega} \mathbf{u}(y) \cdot \nabla_{y}(1 / r) d y=0, \quad x \in \Omega^{\prime} .
$$

For any $\mathbf{u} \in \dot{G}$ we have the following chain of identities

$$
\begin{gathered}
\nabla \int_{\Omega} \mathbf{u}(y) \cdot \nabla_{y}(1 / r) d y=-\nabla \nabla \cdot \int_{\Omega} \mathbf{u}(y)(1 / r) d y=-\nabla \times \nabla \times \int_{\Omega} \mathbf{u}(y)(1 / r) d y \\
=\nabla \times\left[\int_{\Gamma}(1 / r) \mathbf{n}_{y} \times \mathbf{u}(y) d \gamma_{y}-\int_{\Omega}(1 / r) \nabla \times \mathbf{u}(y) d y\right]=0 .
\end{gathered}
$$

Since $\dot{G}$ is dense in $\dot{G}$ and the operator on the left-hand side of (3.18) as a mapping from $G$ into $\mathbf{L}^{2}\left(\Omega^{\prime}\right)$ is bounded (this follows from (3.18)), (3.18) also holds for any $\mathbf{u} \in \stackrel{G}{G}$. This proves the theorem. 
Remark 3.2. In practice one is mostly interested in knowing the field outside the magnetic materal, in $\Omega^{\prime}$. To estimate the error in the approximate solution in $\Omega^{\prime}$, it is therefore required to estimate $\sigma-\sigma_{h}$. In the next section we shall see that when we estimate $\sigma-\sigma_{h}$ directly, we obtain considerably better error estimates (for large permeability) than when we estimate $\sigma-\sigma_{h}$ via $\mathbf{B}-\mathbf{B}_{h}$. The analysis there makes use of the expression (3.14) for $\sigma_{h}$, whereas (3.13) is used for the actual numerical solution.

Remark 3.3. From a finite element point of view, it seems to be a fairly easy task to construct $J_{h}$ in two dimensions, assuming that the stream functions exist. Indeed, standard conforming elements enable us to get directly an approximation $J_{h}$ of $J$ (for $\left.F \in H^{1}, \nabla \times F=\left\{\left(\partial F / \partial x_{2}\right),-\left(\partial F / \partial x_{1}\right)\right\}\right)$. In a three-dimensional problem it is no longer possible to use stream functions to define, in a one-to-one way, a solenoidal vector field. Thus we need to use directly a discretization of $J$ [12] or of $E$ [19] and consider the subspace of solenoidal vector fields.

4. Existence and Uniqueness of Solution. Error Analysis. Assume further throughout that the function $\mathbf{H}(x, \mathbf{B})$ in $(1.1)$ is $1-1$, differentiable with respect to $\mathbf{B}$ for all $x$, measurable in $\Omega$ with respect to $x$ for all $\mathbf{B}$; and for almost all $x \in \Omega$, for all $\mathbf{B} \in R^{3}$ the matrix $\mathbf{H}^{\prime}(\mathbf{B})=\left(\partial H_{i} / \partial B_{j}\right)_{i, j=1,2,3}$ is symmetric (since we neglect hysteresis (see, e.g., [16])) positive definite with its spectrum

$$
\boldsymbol{\sigma}\left((h \mathbf{B})^{\prime}\right) \subset\left[\frac{1}{\mu^{*}}, \frac{1}{\mu_{*}}\right], \quad 1<\mu_{*}<\mu^{*}<\infty .
$$

Remark 4.1. In the isotropic case (1.2), from

$$
\begin{gathered}
\frac{\partial H_{i}}{\partial B_{j}}=\frac{\partial}{\partial B_{j}}\left[\frac{B_{i}}{\mu}\right]=\frac{1}{\mu} \delta_{i j}+\frac{d}{d B}\left[\frac{1}{\mu}\right] \frac{B_{i} B_{j}}{B}, \\
i, j=1,2,3, \delta_{i j}= \begin{cases}1, & i=j, \\
0, & i \neq j,\end{cases} \\
\frac{1}{\mu_{d}} \equiv \frac{d H}{d B}=\frac{d}{d B}\left[\frac{B}{\mu}\right]=\frac{1}{\mu}+B \frac{d}{d B}\left[\frac{1}{\mu}\right]
\end{gathered}
$$

we have

$$
\frac{H_{i}}{B_{j}}=\frac{1}{\mu} \delta_{i k}+\left[\frac{1}{\mu_{d}}-\frac{1}{\mu}\right] \frac{B_{i} B_{j}}{B^{2}} .
$$

If we choose the coordinate system $\mathbf{e}_{1}, \mathbf{e}_{2}, \mathbf{e}_{3}$ in $R^{3}$ so that $\mathbf{e}_{1}$ is parallel to $\mathbf{B}$ then

$$
\mathbf{H}^{\prime}(\mathbf{B})=\left(\begin{array}{ccc}
\frac{1}{\mu_{d}} & 0 & 0 \\
0 & \frac{1}{\mu} & 0 \\
0 & 0 & \frac{1}{\mu}
\end{array}\right) .
$$

In this case the assumption (4.1) is equivalent to

$$
1<\mu_{*} \leqslant \mu, \mu_{d} \leqslant \mu^{*}<\infty \text {. }
$$


LEMMA 4.1. Denote $\mathbf{H}_{i}=h \mathbf{B}_{i}, \mathbf{M}_{i}=\mathbf{B}_{i}-\mathbf{H}_{i}, i=1,2$. We have

$$
\begin{gathered}
\left|\mathbf{M}_{2}-\mathbf{M}_{1}\right| \leqslant\left(1-\frac{1}{\mu^{*}}\right)\left|\mathbf{B}_{2}-\mathbf{B}_{1}\right| \quad \forall \mathbf{B}_{1}, \mathbf{B}_{2} \in R^{3}, \\
\mu_{*}\left|\mathbf{H}_{2}-\mathbf{H}_{1}\right|<\left|\mathbf{B}_{2}-\mathbf{B}_{1}\right| \leqslant \mu^{*}\left|\mathbf{H}_{2}-\mathbf{H}_{1}\right| \quad \forall \mathbf{B}_{1}, \mathbf{B}_{2} \in R^{3} .
\end{gathered}
$$

The operator $R: J \rightarrow J$, defined by (1.6), is strongly monotone with

$$
\left(R \mathbf{B}_{2}-R \mathbf{B}_{1}, \mathbf{B}_{2}-\mathbf{B}_{1}\right) \geqslant \frac{1}{\mu^{*}}\left\|\mathbf{B}_{2}-\mathbf{B}_{1}\right\|^{2} \quad \forall \mathbf{B}_{1}, \mathbf{B}_{2} \in J .
$$

The operator $R: J \rightarrow \mathbf{L}^{2}$ is Lipschitz continuous with

$$
\left\|R \mathbf{B}_{2}-R \mathbf{B}_{1}\right\| \leqslant\left(1+\frac{1}{\mu_{*}}-\frac{1}{\mu^{*}}\right)\left\|\mathbf{B}_{2}-\mathbf{B}_{1}\right\| \quad \forall \mathbf{B}_{1}, \mathbf{B}_{2} \in J .
$$

Proof. Inequalities (4.2) and (4.3) follow from the definitions (1.1) of $H$ and (1.5) of $M,(4.1)$ and the mean value theorem for operators (cf. [23]).

From (1.6) using that by Theorem $2.1\|I-A\|=1$ and the Schwarz inequality,

$$
\begin{aligned}
\left(R \mathbf{B}_{2}-R \mathbf{B}_{1}, \mathbf{B}_{2}-\mathbf{B}_{1}\right) & =\left\|\mathbf{B}_{2}-\mathbf{B}_{1}\right\|^{2}-\left((I-A)\left(\mathbf{M}_{2}-\mathbf{M}_{1}\right), \mathbf{B}_{2}-\mathbf{B}_{1}\right) \\
& \geqslant\left\|\mathbf{B}_{2}-\mathbf{B}_{1}\right\|^{2}-\left\|\mathbf{M}_{2}-\mathbf{M}_{1}\right\|\left\|\mathbf{B}_{2}-\mathbf{B}_{1}\right\| .
\end{aligned}
$$

Now (4.4) follows from (4.2).

Applying the triangle inequality to (1.6) and using that $\|A\|=1,(4.2)$ and (4.3), we arrive at (4.5):

$$
\begin{aligned}
\left\|R \mathbf{B}_{2}-\mathbf{B}_{1}\right\| & \leqslant\left\|\mathbf{H}_{2}-\mathbf{H}_{1}\right\|+\left\|A\left(\mathbf{M}_{2}-\mathbf{M}_{1}\right)\right\| \\
& \leqslant\left(1+\frac{1}{\mu_{*}}-\frac{1}{\mu^{*}}\right)\left\|\mathbf{B}_{2}-\mathbf{B}_{1}\right\| .
\end{aligned}
$$

THEOREM 4.1. For any $\mathbf{B}_{a} \in J$ (1.6) has a unique solution $\mathbf{B} \in J$ and

$$
\|\mathbf{B}\| \leqslant \mu^{*}\left\|\mathbf{B}_{a}-R \mathbf{0}\right\| .
$$

Proof. The assertion of the theorem follows from (4.4) and (4.5) by a standard argument (see, e.g., [27, Theorem 18.5]) from monotone operator theory.

TheOREM 4.2. For any $\mathbf{B}_{a} \in J$ the finite element approximations $\mathbf{B}_{h}$ exist and are unique. Moreover,

$$
\begin{gathered}
\left\|\mathbf{B}_{h}\right\| \leqslant \mu^{*}\left\|P_{J}^{h}\left(\mathbf{B}_{a}-R_{h} \mathbf{0}\right)\right\| \leqslant \mu^{*}\left\|\mathbf{B}_{a}-R_{h} \mathbf{0}\right\|, \\
\left\|\mathbf{B}-\mathbf{B}_{h}\right\| \leqslant \mu^{*}\left(\left\|\left(P_{J}-P_{J}^{h}\right)(I-A) P_{J} \mathbf{M}\right\|+\left\|\left(P_{J}-P_{J}^{h}\right) \mathbf{M}\right\|+\left\|\mathbf{B}_{a}-P_{J}^{h} \mathbf{B}_{a}\right\|\right) .
\end{gathered}
$$

Proof. Rewrite (3.6) and (3.7) in operator form as

$$
\begin{aligned}
R \mathbf{B} & \equiv B-P_{J}(I-A) P_{J} \mathbf{M}=\mathbf{B}_{a}, \quad \mathbf{M}=\mathbf{B}-h \mathbf{B} \equiv \mathbf{B}-\mathbf{H} \\
R_{h} \mathbf{B}_{h} & \equiv \mathbf{B}_{h}-P_{J}^{h}(I-A) P_{J}^{h} \mathbf{M}_{h}=P_{J}^{h} \mathbf{B}_{a}, \quad \mathbf{M}_{h} \equiv \mathbf{B}_{h}-h \mathbf{B}_{h} \equiv \mathbf{B}_{h}-\mathbf{H}_{h},
\end{aligned}
$$


where we used Theorem 2.1. Taking into account that $\left\|P_{J}^{h}(I-A) P_{J}^{h}\right\| \leqslant 1$, in the same way as we used in arriving at (4.4) we obtain

$$
\left(R_{h} \mathbf{B}_{h}-R_{h} \mathbf{0}, \mathbf{B}_{h}\right) \geqslant \frac{1}{\mu^{*}}\left\|\mathbf{B}_{h}\right\|^{2},
$$

and (4.8) follows using Schwarz's inequality.

Subtracting (4.11) from (4.10),

$$
\begin{array}{r}
\mathbf{B}-\mathbf{B}_{h}-P_{J}^{h}(I-A) P_{J}^{h}\left(\mathbf{M}-\mathbf{M}_{h}\right)-P_{J}(I-A) P_{J} \mathbf{M}+P_{J}^{h}(I-A) P_{J}^{h} \mathbf{M} \\
=\mathbf{B}_{a}-P_{J}^{h} \mathbf{B}_{a},
\end{array}
$$

and taking the inner product with $\mathbf{B}-\mathbf{B}_{h}$,

$$
\begin{aligned}
\left\|\mathbf{B}-\mathbf{B}_{h}\right\|^{2}-\left(P_{J}^{h}(I-A) P_{J}^{h}\left(\mathbf{M}-\mathbf{M}_{h}\right), \mathbf{B}-\mathbf{B}_{h}\right) & \\
=\left(\left(P_{J}-P_{J}^{h}\right)(I-A) P_{J} \mathbf{M}-P_{J}^{h}(I-A)\left(P_{J}^{h}-P_{J}\right) \mathbf{M}\right. & \left.\quad+\mathbf{B}_{a}-P_{J}^{h} \mathbf{B}_{a}, \mathbf{B}-\mathbf{B}_{h}\right) .
\end{aligned}
$$

Using that $\left\|P_{J}^{h}(I-A) P_{J}^{h}\right\| \leqslant 1$, (4.2), that $\left\|P_{J}^{h}(I-A)\right\| \leqslant 1$, Schwarz's inequality and the triangle inequality, we arrive at (4.9).

In the next theorem we consider, for simplicity, the isotropic case.

Theorem 4.3. Let $\mathbf{B}_{a} \in U$ and $\mathbf{H}=(1 / \mu) \mathbf{B}$ (see (1.2)). Then for $\boldsymbol{\sigma}$ and $\sigma_{h}$ defined, respectively, by (3.12) and (3.13) we have

$$
\|\boldsymbol{\sigma}\|_{-1 / 2, \Gamma},\left\|\sigma_{h}\right\|_{-1 / 2, \Gamma} \leqslant C\left(\lambda_{0}+\frac{1}{\mu^{*}-1}\right)^{-1}\left\|\mathbf{B}_{a}\right\|, \quad \lambda_{0}>0 .
$$

Suppose, in addition, that the magnetic material is "passive", i.e., $\mu(B)$ satisfies for some $\alpha^{*}$ with $0 \leqslant \alpha^{*} \leqslant \pi / 2$ constant,

(4.1b) $\left(\mathbf{H}_{2}-\mathbf{H}_{1}\right) \cdot\left(\mathbf{M}_{2}-\mathbf{M}_{1}\right) \geqslant \cos \alpha^{*}\left|\mathbf{H}_{2}-\mathbf{H}_{1}\right|\left|\mathbf{M}_{2}-\mathbf{M}_{1}\right| \quad \forall \mathbf{B}_{1}, \mathbf{B}_{2} \in R^{3}$,

and that magnetization is finite (which is always true in practice), i.e., $\mu(B)$ satisfies for some $m>0$,

$$
\|\mathbf{M}\| \equiv\left\|\left(1-\frac{1}{\mu}\right) \mathbf{B}\right\| \leqslant m \quad \forall \mathbf{B} \in J .
$$

Then

$$
\begin{aligned}
& \left\|\boldsymbol{\sigma}-\sigma_{h}\right\|_{-1 / 2, \Gamma} \leqslant 2 C\left(\lambda_{0}+\frac{\cos \alpha^{*}}{\mu^{*}-1}\right)^{-1} \\
& \quad \times\left[\Lambda_{0}\left\|P_{U}\left(P_{J}-P_{J}^{h}\right) \mathbf{M}\right\|\right. \\
& \left.\quad+\sqrt{\Lambda_{0}^{2}\left\|P_{U}\left(P_{J}-P_{J}^{h}\right) \mathbf{M}\right\|^{2}+\frac{8 m}{\mu_{*}-1}\left(\lambda_{0}+\frac{\cos \alpha^{*}}{\mu^{*}-1}\right)\left\|\mathbf{B}-P_{J}^{h} \mathbf{B}\right\|}\right] \\
& +\left\|P_{U}\left(P_{J}-P_{J}^{h}\right) \mathbf{M}\right\|,
\end{aligned}
$$


(4.16) $\left\|\boldsymbol{\sigma}-\sigma_{h}\right\|_{-1 / 2, \Gamma} \leqslant C\left(\left\|\left(P_{J}-P_{J}^{h}\right) \mathbf{M}\right\|+\left(1-\frac{1}{\mu^{*}}\right)\left\|\mathbf{B}-P_{J}^{h} \mathbf{B}\right\|\right)$

$\times\left[1+\frac{2}{\sqrt{\left(\frac{1}{\mu^{*}-1}\right)^{2}+\frac{\lambda_{0}}{m}\left(\left\|\left(P_{J}-P_{J}^{h}\right) \mathbf{M}\right\|+\left(1-\frac{1}{\mu^{*}}\right)\left\|\mathbf{B}-P_{J}^{h} \mathbf{B}\right\|\right)+\frac{1}{\mu^{*}-1}}}\right]$

Proof. Using that by Theorem $2.1 \operatorname{Ker} A=j$, rewrite (3.6) and (3.7), respectively, as

$$
\begin{gathered}
\left(\mathbf{H}+P_{U} A P_{U} \mathbf{M}-\mathbf{B}_{a}, \mathbf{u}\right)=0 \quad \forall \mathbf{u} \in J, \\
\left(\mathbf{H}_{h}+P_{U} A P_{U} P_{J}^{h} \mathbf{M}_{h}-\mathbf{B}_{a}, \mathbf{\chi}\right)=0 \quad \forall \mathbf{\chi} \in J_{h} .
\end{gathered}
$$

Choosing in (4.17) $\mathbf{u}=P_{J} \mathbf{M}$ and using Theorem 2.1, (4.2) and (4.3),

$$
\begin{aligned}
\left\|\mathbf{B}_{a}\right\|\left\|P_{U} \mathbf{M}\right\| & \geqslant\left(\mathbf{B}_{a}, P_{U} \mathbf{M}\right)=\left(\mathbf{B}_{a}, P_{J} \mathbf{M}\right)=\left(\mathbf{H}, P_{J} \mathbf{M}\right)+\left(A P_{U} \mathbf{M}, P_{U} \mathbf{M}\right) \\
& \geqslant(\mathbf{H}, \mathbf{B})-\left\|P_{J} \mathbf{H}\right\|^{2}+\lambda_{0}\left\|P_{U} \mathbf{M}\right\|^{2} \geqslant(\mathbf{H}, \mathbf{M})+\lambda_{0}\left\|P_{U} \mathbf{M}\right\|^{2} \\
& \geqslant \frac{1}{\mu^{*}-1}\|\mathbf{M}\|^{2}+\lambda_{0}\left\|P_{U} \mathbf{M}\right\|^{2} \geqslant\left(\frac{1}{\mu^{*}-1}+\lambda_{0}\right)\left\|P_{U} \mathbf{M}\right\|^{2} .
\end{aligned}
$$

Taking into account Lemma 2.1, we get from (4.19) the first part of (4.14). Choosing $\mathrm{X}=P_{J}^{h} \mathbf{M}_{h}$ in (4.18) we derive the second part in the same way:

$$
\begin{aligned}
& \left\|\mathbf{B}_{a}\right\|\left\|P_{U} P_{J}^{h} \mathbf{M}_{h}\right\| \geqslant\left(\mathbf{B}_{a}, P_{U} P_{J}^{h} \mathbf{M}_{h}\right)=\left(\mathbf{B}_{a}, P_{J}^{h} \mathbf{M}_{h}\right) \\
& \quad=\left(\mathbf{H}_{h}, P_{J}^{h} \mathbf{M}_{h}\right)+\left(A P_{U} P_{J}^{h} \mathbf{M}_{h}, P_{U} P_{J}^{h} \mathbf{M}_{h}\right) \\
& \quad \geqslant\left(\mathbf{H}_{h}, \mathbf{B}_{h}\right)-\left\|P_{J}^{h} \mathbf{H}_{h}\right\|^{2}+\lambda_{0}\left\|P_{U} P_{J}^{h} \mathbf{M}_{h}\right\|^{2} \\
& \quad \geqslant\left(\mathbf{H}_{h}, \mathbf{M}_{h}\right)+\lambda_{0}\left\|P_{U} P_{J}^{h} \mathbf{M}_{h}\right\|^{2} \geqslant\left(\lambda_{0}+\frac{1}{\mu^{*}-1}\right)\left\|P_{U} P_{J}^{h} \mathbf{M}_{h}\right\|^{2} .
\end{aligned}
$$

To derive (4.16), we choose $\mathbf{u}=P_{J}^{h}\left(\mathbf{M}-\mathbf{M}_{h}\right), \chi=\mathbf{u}$ and subtract (4.18) from (4.17) to obtain

$$
\begin{aligned}
I_{1}+I_{2} & \equiv\left(\mathbf{H}-\mathbf{H}_{h}, P_{J}^{h}\left(\mathbf{M}-\mathbf{M}_{h}\right)\right)+\left(A P_{U} P_{J}^{h}\left(\mathbf{M}-\mathbf{M}_{h}\right), P_{J}^{h}\left(\mathbf{M}-\mathbf{M}_{h}\right)\right) \\
& =\left(A P_{U}\left(P_{J}^{h}-P_{J}\right) \mathbf{M}, P_{U} P_{J}^{h}\left(\mathbf{M}-\mathbf{M}_{h}\right)\right) .
\end{aligned}
$$

Proceeding as above and using (4.1b), (4.2), (4.3) and (4.1c),

$$
\begin{gathered}
I_{1}=\left(\mathbf{H}-\mathbf{H}_{h}, \mathbf{B}-\mathbf{B}_{h}\right)-\left\|P_{J}^{h}\left(\mathbf{H}-\mathbf{H}_{h}\right)\right\|^{2}+\left(\mathbf{H}-\mathbf{H}_{h}, P_{J}^{h} \mathbf{B}-\mathbf{B}\right) \\
\geqslant\left(\mathbf{H}-\mathbf{H}_{h}, \mathbf{M}-\mathbf{M}_{h}\right)+\left(\mathbf{H}-\mathbf{H}_{h}, P_{J}^{h} \mathbf{B}-\mathbf{B}\right) \\
\geqslant \cos \alpha^{*}\left(\left|\mathbf{H}-\mathbf{H}_{h}\right|,\left|\mathbf{M}-\mathbf{M}_{h}\right|\right)-\left\|\mathbf{H}-\mathbf{H}_{h}\right\|\left\|\mathbf{B}-P_{J}^{h} \mathbf{B}\right\| \\
\geqslant \frac{\cos \alpha^{*}}{\mu^{*}-1}\left\|\mathbf{M}-\mathbf{M}_{h}\right\|^{2}-\frac{1}{\mu_{*}-1}\left\|\mathbf{M}-\mathbf{M}_{h}\right\|\left\|\mathbf{B}-P_{J}^{h} \mathbf{B}\right\| \\
\geqslant \frac{\cos \alpha^{*}}{\mu^{*}-1}\left\|P_{U} P_{J}^{h}\left(\mathbf{M}-\mathbf{M}_{h}\right)\right\|^{2}-\frac{2 m}{\mu_{*}-1}\left\|\mathbf{B}-P_{J}^{h} \mathbf{B}\right\|, \\
I_{2} \geqslant \lambda_{0}\left\|P_{U} P_{J}^{h}\left(\mathbf{M}-\mathbf{M}_{h}\right)\right\|^{2} .
\end{gathered}
$$


Substituting (4.22) and (4.23) into (4.21) and using Theorem 2.1,

$$
\begin{aligned}
\left(\lambda_{0}+\right. & \left.\frac{\cos \alpha^{*}}{\mu^{*}-1}\right)\left\|P_{U} P_{J}^{h}\left(\mathbf{M}-\mathbf{M}_{h}\right)\right\|^{2} \\
& -\Lambda_{0}\left\|P_{U}\left(P_{J}^{h}-P_{J}\right) \mathbf{M}\right\|\left\|P_{U} P_{J}\left(\mathbf{M}-\mathbf{M}_{h}\right)\right\|-\frac{2 m}{\mu_{*}-1}\left\|\mathbf{B}-P_{J}^{h} \mathbf{B}\right\| \leqslant 0 .
\end{aligned}
$$

And (4.24) implies (since $a x^{2}-b x-c \leqslant 0, a, b, c>0$ implies, completing the squares, $\left.x \leqslant\left(b+\sqrt{b^{2}+4 a c}\right) / 2 a\right)$,

$$
\begin{aligned}
& \left\|P_{U} P_{J}^{h}\left(\mathbf{M}-\mathbf{M}_{h}\right)\right\| \\
& \leqslant \frac{\sqrt{\lambda_{0}^{2}\left\|P_{U}\left(P_{J}-P_{J}^{h}\right) \mathbf{M}\right\|^{2}+\frac{8 m}{\mu_{*}-1}\left(\lambda_{0}+\frac{\cos \alpha^{*}}{\mu^{*}-1}\right)\left\|\mathbf{B}-P_{J}^{h} \mathbf{B}\right\|}}{2\left(\lambda_{0}+\frac{\cos \alpha^{*}}{\mu^{*}-1}\right)} .
\end{aligned}
$$

And (4.15) follows from (4.25), (3.12), (3.14), Lemma 2.1, and

$$
\left\|P_{U}\left(\mathbf{M}-P_{J}^{h} \mathbf{M}_{h}\right)\right\| \leqslant\left\|P_{U} P_{J}^{h}\left(\mathbf{M}-\mathbf{M}_{h}\right)\right\|+\left\|P_{U}\left(P_{J}-P_{J}^{h}\right) \mathbf{M}\right\| .
$$

We proceed now to prove (4.16). Setting $\mathbf{B}^{h}=P_{J}^{h} \mathbf{B}, \mathbf{M}^{h}=\mathbf{B}^{h}-h \mathbf{B}^{h}$ and subtracting (4.11) from (4.10) projected onto $J_{h}$,

$$
\mathbf{0}=\mathbf{B}^{h}-\mathbf{B}_{h}-P_{J}^{h}(I-A) P_{J} \mathbf{M}+P_{J}^{h}(I-A) P_{J}^{h} \mathbf{M}_{h} .
$$

Taking the inner product with $\mathbf{B}^{h}-\mathbf{B}_{h}$,

$$
\begin{gathered}
\left\|\mathbf{B}^{h}-\mathbf{B}_{h}\right\|^{2}-\left(P_{J}^{h}(I-A) P_{J}^{h}\left(\mathbf{M}^{h}-\mathbf{M}_{h}\right), \mathbf{B}^{h}-\mathbf{B}_{h}\right) \\
=\left(P_{J}^{h}(I-A)\left(P_{J} \mathbf{M}-P_{J}^{h} \mathbf{M}^{h}\right), \mathbf{B}^{h}-\mathbf{B}_{h}\right) .
\end{gathered}
$$

Using the Schwarz inequality, and that $\left\|P_{J}^{h}(I-A)\right\| \leqslant 1$,

$$
I \equiv\left\|\mathbf{B}^{h}-\mathbf{B}_{h}\right\|-\left\|P_{J}^{h}(I-A) P_{J}^{h}\left(\mathbf{M}-\mathbf{M}_{h}\right)\right\| \leqslant\left\|P_{J} \mathbf{M}-P_{J}^{h} \mathbf{M}^{h}\right\| .
$$

Using (4.2), the notation $\mathbf{u}=P_{J}^{h}\left(\mathbf{M}^{h}-\mathbf{M}_{h}\right)$, Theorem 2.1, the inequality

$$
\begin{aligned}
\sqrt{a^{2}+b^{2}} & -\sqrt{a^{2}+\left(1-\lambda_{0}\right)^{2} b^{2}} \\
& =\frac{\left[1-\left(1-\lambda_{0}\right)^{2}\right] b^{2}}{\sqrt{a^{2}+b^{2}}+\sqrt{a^{2}+\left(1-\lambda_{0}\right)^{2} b^{2}}} \geqslant \frac{\lambda_{0} b^{2}}{2 \sqrt{a^{2}+b^{2}}}
\end{aligned}
$$

with $a=\left\|P_{j} \mathbf{u}\right\|, b=\left\|P_{U} \mathbf{u}\right\|$, and (4.1c),

$$
\begin{gathered}
I \geqslant \frac{\mu^{*}}{\mu^{*}-1}\|\mathbf{u}\|-\|(I-A) \mathbf{u}\| \geqslant \frac{1}{\mu^{*}-1}\|\mathbf{u}\|+\sqrt{\left\|P_{j} \mathbf{u}\right\|^{2}+\left\|P_{U} \mathbf{u}\right\|^{2}} \\
-\left\|P_{j} \mathbf{u}\right\|^{2}+\left\|(I-A) P_{U} \mathbf{u}\right\|^{2} \geqslant \frac{1}{\mu^{*}-1}\|\mathbf{u}\|+\frac{\lambda_{0}\left\|P_{U} \mathbf{u}\right\|^{2}}{2\|\mathbf{u}\|} .
\end{gathered}
$$

Since $\left\|P_{U} \mathbf{u}\right\| \leqslant\|\mathbf{u}\|$ and by (4.1c) $\|\mathbf{u}\| \leqslant 2 m$,

$$
I \leqslant \frac{1}{\mu^{*}-1}\left\|P_{U} \mathbf{u}\right\|+\frac{\lambda_{0}}{4 m}\left\|P_{U} \mathbf{u}\right\|^{2} .
$$


And therefore, completing the squares,

$$
\begin{aligned}
\left\|P_{U} P_{J}^{h}\left(\mathbf{M}^{h}-\mathbf{M}_{h}\right)\right\| & \leqslant\left(\sqrt{\left.\left(\frac{1}{\mu^{*}-1}\right)^{2}+\frac{\lambda_{0}}{m} I-\frac{1}{\mu^{*}-1}\right)} \frac{2 m}{\lambda_{0}}\right. \\
& =\frac{2 I}{\sqrt{\left(\frac{1}{\mu^{*}-1}\right)^{2}+\frac{\lambda_{0}}{m} I+\frac{1}{\mu^{*}-1}}} \equiv \phi .
\end{aligned}
$$

We now estimate $\phi(I)$ using that $\phi(x)$ is monotone increasing for $x \geqslant 0$. From (4.27)

$$
\begin{aligned}
I & \leqslant\left\|P_{J} \mathbf{M}-P_{J}^{h} \mathbf{M}^{h}\right\| \leqslant\left\|\left(P_{J}-P_{J}^{h}\right) \mathbf{M}\right\|+\left\|P_{J}^{h}\left(\mathbf{M}-\mathbf{M}^{h}\right)\right\| \\
& \leqslant\left\|\left(P_{J}-P_{J}^{h}\right) \mathbf{M}\right\|+\left(1-\frac{1}{\mu^{*}}\right)\left\|\mathbf{B}-\mathbf{B}^{h}\right\| .
\end{aligned}
$$

It follows that

$$
\begin{aligned}
& \left\|P_{U} P_{J}^{h}\left(\mathbf{M}^{h}-\mathbf{M}_{h}\right)\right\| \\
& \leqslant \frac{2\left(\left\|\left(P_{J}-P_{J}^{h}\right) \mathbf{M}\right\|+\left(1-\frac{1}{\mu^{*}}\right)\left\|\mathbf{B}-\mathbf{B}^{h}\right\|\right)}{\sqrt{\left(\frac{1}{\mu^{*}-1}\right)^{2}+\frac{\lambda_{0}}{m}\left\|\left(P_{J}-P_{J}^{h}\right) \mathbf{M}\right\|+\left(1-\frac{1}{\mu^{*}}\right)\left\|\mathbf{B}-\mathbf{B}^{h}\right\|+\frac{1}{\mu^{*}-1}}} .
\end{aligned}
$$

Using the triangle inequality and (4.2),

$$
\begin{aligned}
\| P_{U}(\mathbf{M} & \left.-P_{J}^{h} \mathbf{M}_{h}\right) \| \\
& \leqslant\left\|\left(P_{J}-P_{J}^{h}\right) \mathbf{M}\right\|+\left\|P_{J}^{h}\left(\mathbf{M}-\mathbf{M}^{h}\right)\right\|+\left\|P_{U} P_{J}^{h}\left(\mathbf{M}^{h}-\mathbf{M}_{h}\right)\right\| \\
& \leqslant\left\|\left(P_{J}-P_{J}^{h}\right) \mathbf{M}\right\|+\left(1-\frac{1}{\mu^{*}}\right)\left\|\mathbf{B}-\mathbf{B}^{h}\right\|+\left\|P_{U} P_{J}^{h}\left(\mathbf{M}^{h}-\mathbf{M}_{h}\right)\right\| .
\end{aligned}
$$

Substituting (4.29) into (4.30), using (3.12), (3.14) and Lemma 2.1, we arrive finally at (4.16).

Remark 4.2. Using the estimate (4.9) one can easily obtain an estimate for $\sigma-\sigma_{h}$. This estimate, including $\mu^{*}$ as a multiple, is appropriate when $\mu^{*}$ is not large but deteriorates when $\mu^{*} \rightarrow \infty$. The estimates (4.15) and (4.16) are more accurate. They tend to a finite limit when $\mu^{*} \rightarrow \infty$ : (4.15) is quasi optimal in the limiting case $\mu_{*} \rightarrow \infty$ (and therefore $\mu^{*} \rightarrow \infty$ ) and (4.16) is quasi optimal when $\mu^{*}$ is finite. We have not been able to obtain an optimal estimate in the limiting case $\mu^{*} \rightarrow \infty$, and $\mu_{*}$ is finite. The difficulties with the estimation of $\mathbf{H}-\mathbf{H}_{h}$ arise because $\mathbf{H}_{h} \notin G$, while $\mathbf{H} \in G$, i.e., $P_{j} \mathbf{H} \neq \mathbf{0}$.

5. Solution by Successive Iteration. Consider the successive iteration method

$$
\mathbf{B}_{n+1}=(I-A) P_{J} \mathbf{M}_{n}+\mathbf{B}_{a} \equiv K\left(\mathbf{B}_{n}\right)+\mathbf{B}_{a}, \quad n=0,1, \ldots,
$$

of solution of (3.6), where $\mathbf{M}_{n}=\mathbf{B}_{n}-h \mathbf{B}_{n}$. Note that Theorem 5.1 below is also valid for $\mathbf{B}$ replaced by $\mathbf{B}_{h}$, where the corresponding iteration method of solution of 
(3.7) is given by

$$
\begin{aligned}
\left(\left(\mathbf{B}_{h}\right)_{n+1}, \mathbf{u}\right)=\left(\left(\mathbf{B}_{h}\right)_{n}-h\left(\mathbf{B}_{h}\right)_{n}-A\left(\left(\mathbf{B}_{h}\right)_{n}-P_{J}^{h} h\left(\mathbf{B}_{h}\right)_{n}\right)+\mathbf{B}_{a}, \mathbf{u}\right) & \\
& \forall \mathbf{u} \in J_{h} .
\end{aligned}
$$

THEOREM 5.1. Let $h$ satisfy (4.1). Then the operator $K$ in $J$ defined by (5.1) is Lipschitz continuous with

$$
\left\|K\left(\mathbf{B}_{2}\right)-K\left(\mathbf{B}_{1}\right)\right\| \leqslant\left(1-\frac{1}{\mu^{*}}\right)\left\|\mathbf{B}_{2}-\mathbf{B}_{1}\right\| \quad \forall \mathbf{B}_{1}, \mathbf{B}_{2} \in J
$$

Moreover, the successive iterations (5.1) converge to the solution B of (3.6) and

$$
\left\|\mathbf{B}-\mathbf{B}_{n}\right\| \leqslant \mu^{*}\left(1-\frac{1}{\mu^{*}}\right)^{n}\left\|\mathbf{B}_{1}-\mathbf{B}_{0}\right\| .
$$

Proof. Inequality (5.2) follows from (4.2) and the fact that by Theorem 2.1 $\|I-A\|=1$; (5.3) follows from (5.2).

Recall that we are mostly interested in knowing the solution $\mathbf{B}$ outside of the magnetic material. We therefore would like to know the rate of convergence of $P_{U} \mathbf{M}_{n}$. From (4.2), (5.3) and the fact that $\left\|P_{U}\right\|=1$ we have

Corollary 5.1. For $\mathbf{M}=\mathbf{B}-h \mathbf{B}$ we have

$$
\left\|P_{U}\left(\mathbf{M}-\mathbf{M}_{n}\right)\right\| \leqslant\left(1-\frac{1}{\mu^{*}}\right)\left\|\mathbf{B}-\mathbf{B}_{n}\right\| \leqslant \mu^{*}\left(1-\frac{1}{\mu^{*}}\right)^{n+1}\left\|\mathbf{B}_{1}-\mathbf{B}_{0}\right\| .
$$

We shall now study more closely the convergence of $P_{U} \mathbf{M}_{n}$ in the case of large $\mu$. It is convenient to introduce a function

$$
\varepsilon(B)=1-\mu_{*} / \mu .
$$

Then $\varepsilon(B) \mathbf{B}=\mathbf{B}-\mu_{*} \mathbf{H}$, and by (4.1a)

$$
0 \leqslant \varepsilon(B) \leqslant 1, \quad\left\|(\varepsilon(B) \mathbf{B})^{\prime}\right\|_{R^{3}} \leqslant \varepsilon^{*} \equiv 1-\mu_{*} / \mu^{*},
$$

where the notation $\|\cdot\|_{R^{3}}$ stands for the Euclidean matrix norm.

TheOREM 5.2. Let $\mathbf{M}=\left(1-(1-\varepsilon(B)) / \mu^{*}\right) \mathbf{B}$ and $\varepsilon(B)$ satisfy (5.5). Then under the assumptions of Theorem 5.1 we have

$$
\begin{aligned}
&\left\|P_{U}\left(\mathbf{M}_{n+1}-\mathbf{M}\right)\right\| \\
& \leqslant \frac{1}{\mu_{*}}\left(1-\frac{1}{\mu^{*}}\right)^{n+1}\left(1-\frac{\mu_{*}}{\mu^{*}}\right)\left(1+\frac{1}{\mu_{*}}\right) \\
& \times\left[\left(1-\lambda_{0}\right)^{n+1}\left(1-\frac{1}{\mu^{*}}\right) \frac{\mu^{*}}{1+\lambda_{0}\left(\mu^{*}-1\right)}+\frac{1}{\lambda_{0}}\left(\frac{\mu^{*}}{\mu_{*}}-1\right)\right]\left\|\mathbf{B}_{0}\right\|,
\end{aligned}
$$

where $\mathbf{B}_{0}$ solves

$$
\frac{1}{\mu_{*}} \mathbf{B}_{0}+\left(1-\frac{1}{\mu_{*}}\right) A \mathbf{B}_{0}=\mathbf{B}_{a}
$$


Proof. We shall use the notation $\varepsilon=\varepsilon(B), \varepsilon_{n}=\varepsilon\left(B_{n}\right)$. Using (5.5), that $\left\|P_{U}\right\|=1$ and that for $\mathbf{u} \in J, \mathbf{u}=P_{U} \mathbf{u}+P_{j} \mathbf{u}$,

$$
\begin{aligned}
& \left\|P_{U}\left(\mathbf{M}_{n+1}-\mathbf{M}\right)\right\|=\left\|P_{U}\left[\left(1-\frac{1-\varepsilon_{n+1}}{\mu_{*}}\right)\right] \mathbf{B}_{n+1}-\left(1-\frac{1-\varepsilon}{\mu_{*}}\right) \mathbf{B}\right\| \\
& \quad \leqslant\left(1-\frac{1}{\mu_{*}}\right)\left\|P_{U}\left(\mathbf{B}_{n+1}-\mathbf{B}\right)\right\|+\frac{1}{\mu_{*}}\left\|P_{U}\left(\varepsilon_{n+1} \mathbf{B}_{n+1}-\varepsilon \mathbf{B}\right)\right\| \\
& \quad \leqslant\left(1-\frac{1}{\mu_{*}}\right)\left\|P_{U}\left(\mathbf{B}_{n+1}-\mathbf{B}\right)\right\|+\frac{\varepsilon^{*}}{\mu_{*}}\left\|\mathbf{B}_{n+1}-\mathbf{B}\right\| \\
& \quad \leqslant\left(1-\frac{1}{\mu^{*}}\right)\left\|P_{U}\left(\mathbf{B}_{n+1}-\mathbf{B}\right)\right\|+\frac{\varepsilon^{*}}{\mu_{*}}\left\|P_{J}\left(\mathbf{B}_{n+1}-\mathbf{B}\right)\right\| .
\end{aligned}
$$

We proceed now to estimate the first term in (5.8). Denote, for convenience,

$$
a_{n}=\left\|P_{U}\left(\mathbf{B}_{n}-\mathbf{B}_{n-1}\right)\right\|, \quad b_{n}=\left\|\mathbf{B}_{n}-\mathbf{B}_{n-1}\right\| .
$$

From (5.1), (5.5), using that by Theorem $2.1\left\|P_{U}(I-A)\right\|=1-\lambda_{0}$, (5.2),

$$
\begin{aligned}
& a_{n+1}=\left\|P_{U}(I-A)\left[\left(1-\frac{1-\varepsilon_{n}}{\mu_{*}}\right) \mathbf{B}_{n}-\left(1-\frac{1-\varepsilon_{n-1}}{\mu_{*}}\right) \mathbf{B}_{n-1}\right]\right\| \\
& \quad \leqslant\left(1-\lambda_{0}\right)\left[\left(1-\frac{1}{\mu_{*}}\right) a_{n}+\frac{\varepsilon^{*}}{\mu_{*}} b_{n}\right] \\
& \quad \leqslant\left(1-\lambda_{0}\right)\left[\left(1-\frac{1}{\mu_{*}}\right) a_{n}+\frac{\varepsilon^{*}}{\mu_{*}}\left(1-\frac{1}{\mu^{*}}\right)^{n-1} b_{1}\right] \\
& \quad \leqslant\left(1-\lambda_{0}\right)\left\{\left(1-\frac{1}{\mu^{*}}\right)\left(1-\lambda_{0}\right)\left[\left(1-\frac{1}{\mu^{*}}\right) a_{n-1}+\frac{\varepsilon^{*}}{\mu_{*}}\left(1-\frac{1}{\mu^{*}}\right)^{n-2} b_{1}\right]\right. \\
& \quad=\left(1-\lambda_{0}\right)^{2}\left(1-\frac{1}{\mu_{*}^{*}}\left(1-\frac{1}{\mu^{*}}\right)^{n-1} b_{1}\right\} \\
& \leqslant\left(1-\lambda_{0}\right)^{n} \times\left(1-\frac{1}{\mu^{*}}\right)^{n} a_{1}+\frac{\varepsilon^{*}}{\mu_{*}}\left(1-\frac{1}{\mu^{*}}\right)^{n-1}\left(1-\lambda_{0}\right) b_{1}\left[1+\left(1-\lambda_{0}\right)\right] \\
& \quad=\left(1-\lambda_{0}\right)^{n}\left(1-\frac{1}{\mu^{*}}\right)^{n-1} a_{1}+\frac{\varepsilon^{*}}{\mu_{*}}\left(1-\frac{1}{\mu^{*}}\right)^{n-1} b_{1} \frac{\left(1-\lambda_{0}\right) b_{1} \sum_{i=0}^{n-1}\left(1-\lambda_{0}\right)^{i}}{\lambda_{0}} \\
& \leqslant\left(1-\lambda_{0}\right)^{n}\left(1-\frac{1}{\mu^{*}}\right)^{n} a_{1}+\frac{\left.\left.1-\lambda_{0}\right)^{n}\right]}{\lambda_{0}} \frac{\varepsilon^{*}}{\mu_{*}}\left(1-\frac{1}{\mu^{*}}\right)^{n-1} b_{1} .
\end{aligned}
$$

If $m>n$ it follows that

$$
\begin{aligned}
\left\|P_{U}\left(\mathbf{B}_{n}-\mathbf{B}_{m}\right)\right\| \leqslant & \sum_{i=n+1}^{m} a_{i} \leqslant\left(1-\lambda_{0}\right)^{n}\left(1-\frac{1}{\mu^{*}}\right)^{n} \times \frac{1}{1-\left(1-\lambda_{0}\right)\left(1-\frac{1}{\mu^{*}}\right)} a_{1} \\
& +\left(1-\frac{1}{\mu^{*}}\right)^{n-1} \frac{1-\lambda_{0}}{\lambda_{0}} \frac{\varepsilon^{*}}{\mu_{*}} \frac{1}{1-\left(1-\frac{1}{\mu^{*}}\right)} b_{1} .
\end{aligned}
$$


Passing to the limit when $m \rightarrow \infty$, we get

$$
\begin{aligned}
\left\|P_{U}\left(\mathbf{B}_{n+1}-\mathbf{B}\right)\right\| \leqslant\left(1-\frac{1}{\mu^{*}}\right)^{n}[(1 & \left.-\lambda_{0}\right)^{n+1}\left(1-\frac{1}{\mu^{*}}\right) \\
& \left.\times \frac{\mu^{*}}{1+\lambda_{0}\left(\mu^{*}-1\right)} a_{1}+\frac{1-\lambda_{0}}{\lambda_{0}} \frac{\mu^{*}}{\mu_{*}} \varepsilon^{*} b_{1}\right] .
\end{aligned}
$$

Substituting (5.9) and (5.8) and using Theorem 5.1 and (5.5),

$$
\begin{aligned}
& \left\|P_{U}\left(\mathbf{M}_{n+1}-\mathbf{M}\right)\right\| \\
& \leqslant\left(1-\frac{1}{\mu^{*}}\right)^{n+1}\left[\left(1-\lambda_{0}\right)^{n+1}\left(1-\frac{1}{\mu^{*}}\right) \frac{\mu^{*}}{1+\lambda_{0}\left(\mu^{*}-1\right)}\right] \\
& \times\left\|P_{U}\left(\mathbf{B}_{1}-\mathbf{B}_{0}\right)\right\|+\frac{1-\lambda_{0}}{\lambda_{0}} \frac{\mu^{*}}{\mu_{*}} \varepsilon^{*}\left\|\mathbf{B}_{1}-\mathbf{B}_{0}\right\|+\frac{\mu^{*}}{\mu_{*}} \varepsilon^{*}\left(1-\frac{1}{\mu^{*}}\right)^{n+1} \\
& \times\left\|\mathbf{B}_{1}-\mathbf{B}_{0}\right\| \\
& \leqslant\left(1-\frac{1}{\mu^{*}}\right)^{n+1}\left\{\left(1-\lambda_{0}\right)^{n+1}\left(1-\frac{1}{\mu^{*}}\right) \frac{\mu^{*}}{1+\lambda_{0}\left(\mu^{*}-1\right)}\right. \\
& \times\left\|P_{U}\left[\frac{1-\varepsilon}{\mu_{*}} B_{0}+A P_{J}\left(1-\frac{1-\varepsilon}{\mu^{*}}\right) \mathbf{B}_{0}-\mathbf{B}_{a}\right]\right\| \\
& +\frac{1}{\lambda_{0}} \frac{\mu^{*}}{\mu_{*}}\left(1-\frac{\mu_{*}}{\mu^{*}}\right) \\
& \left.\times\left\|P_{J}\left[\left(\frac{1-\varepsilon}{\mu_{*}}\right) \mathbf{B}_{0}+A P_{J}\left(1-\frac{1-\varepsilon}{\mu_{*}}\right) \mathbf{B}_{0}-\mathbf{B}_{a}\right]\right\|\right\} \\
& \leqslant\left(1-\frac{1}{\mu^{*}}\right)^{n+1} \times\left[\left(1-\lambda_{0}\right)^{n+1}\left(1-\frac{1}{\mu^{*}}\right) \frac{\mu^{*}}{1+\lambda_{0}\left(\mu^{*}-1\right)}+\frac{1}{\lambda_{0}}\left(\frac{\mu^{*}}{\mu_{*}}-1\right)\right] \\
& \times\left\{\left\|P_{J}\left[\frac{1}{\mu_{*}} \mathbf{B}_{0}+\left(1-\frac{1}{\mu_{*}}\right) A \mathbf{B}_{0}-\mathbf{B}_{a}\right]\right\|+\frac{1}{\mu_{*}}\left\|P_{J}\left(\varepsilon \mathbf{B}_{0}+\frac{1}{\mu_{*}} A P_{J} \varepsilon \mathbf{B}_{0}\right)\right\|\right\} .
\end{aligned}
$$

Now (5.6) follows from (5.10), (5.7) and the estimate

$$
\left\|P_{J}\left(\varepsilon \mathbf{B}_{0}+\frac{1}{\mu_{*}} A P_{J} \varepsilon \mathbf{B}_{0}\right)\right\| \leqslant\left(\varepsilon^{*}+\frac{\varepsilon^{*}}{\mu_{*}}\right)\left\|\mathbf{B}_{0}\right\|=\left(1-\frac{\mu_{*}}{\mu^{*}}\right)\left(1+\frac{1}{\mu_{*}}\right)\left\|\mathbf{B}_{0}\right\| .
$$

Remark 5.1. Comparing the estimates (5.4) and (5.6) we observe that for small $\mu$ they are comparable. However for large $\mu$ (which is often the case in practice) (5.6) is much better due to appropriate choice (5.7) of $\mathbf{B}_{0}$ and the greater use of properties of $A$.

Acknowledgments. The author wishes to thank Professor James H. Bramble of Cornell University for his encouragement and guidance during the preparation of the dissertation from which these results have been taken. The author also wants to express his gratitude to Professor Lars B. Wahlbin of Cornell University for clarifying discussions and his suggestions concerning the presentation.

Department of Mathematics Iowa State University

Ames, Iowa 50011 
1. P. G. Akishin, S. B. Vorozhtsoz \& E. P. Zhidkov, Calculation of the Magnetic Field of the Isochronous Cyclotron Sector Magnet by the Integral Equation Method, Proc. Compumag Conf., Grenoble, 1978.

2. A. G. Armstrong, A. M. Collie, C. J. Diserens, N. J. Newman, M. Simkin \& C. W. Trowbridge, New Developments in the Magnet Design Program GFUN, Rutherford Laboratory Report No. RL-5060.

3. J. H. BRamble \& J. E. Pasciak, "A new computational approach for the linearized scalar potential formulation of the magnetostatic field problem," IEEE Trans. Mag., v. Mag-18, 1982, pp. 357-361.

4. M. V. K. ChARI \& P. P. Silvester (editors), Finite Elements in Electrical and Magnetic Field Problems, Wiley, New York, 1978.

5. M. J. Friedman, "Mathematical study of the nonlinear singular integral magnetic field equation 1," SIAM J. Appl. Math., v. 44, 1980, pp. 14-20.

6. M. J. Friedman, "Mathematical study of the nonlinear singular integral magnetic field equation 2," SIAM J. Numer. Anal., v. 18, 1981, pp. 644-653.

7. M. J. Friedman, "Mathematical study of the nonlinear singular integral magnetic field equation 3," SIAM J. Math. Anal., v. 12, 1981, pp. 536-540.

8. M. J. FRIEDMAN \& J. S. Colonias, "On the coupled differential-integral equations for the solution of the general magnetostatic problem," IEEE Trans. Mag., v. Mag-18, No. 2, March 1982, pp. 336-339.

9. M. J. Friedman, Finite Element Formulation of the General Magnetostatic Problem in the Space of Solenoidal Vector Functions, Ph.D. Thesis, Cornell University, 1982.

10. M. J. Friedman \& J. E. Pasciak, "Spectral properties for the magnetization integral operator," Math. Comp., v. 43, 1984, pp. 447-453.

11. R. Glowinski \& A. MARRocco, "Numerical solution of two-dimensional magnetostatic problems by augmented Lagrangian methods,” Comput. Methods Appl. Mech. Engrg., v. 12, 1977, pp. 33-46.

12. F. HECHT, "Construction d'une base de fonctions $P_{1}$ non conforme à divergence nulle dans $\mathbf{R}^{3}$," RAIRO Anal. Numér., v. 15, 1981, pp. 119-150.

13. J. L. Lions \& E. Magenes, Nonhomogeneous Boundary Value Problems and Applications, Vol. 1, Springer-Verlag, New York, 1972.

14. M. H. LeAN \& A. WeXler, Accurate Field Computation with the Boundary Element Method, Proc. Compumag Conf., Chicago, 1982.

15. B. H. MCDONALD \& W. WeXLER, "Mutually constrained partial differential and integral equation field formulation," in Finite Elements in Electrical and Magnetic Field Problems (M. V. U. Chari and P. Silvester, eds.), Wiley, New York, 1978.

16. I. D. MAYergoyz, Iterational Methods for the Computation of the Static Fields in Nonhomogeneous, Anisotropic and Nonlinear Medias, Naukova Dumka, Kiev, 1979. (Russian)

17. I. D. MAYERGOYZ, "On numerical investigation of magnetostatic field in nonlinear ferromagnetic media," Sbornik Kibernetika, Vychislitel'naya Tekhnika, v. 17, 1972. (Russian)

18. J. NeCAS, Les Méthodes Directes en Théorie des Équations Elliptiques, Masson, Paris, 1967.

19. J. C. NedELEC, Mixed Finite Elements in $R^{3}$, Rapport Interne no. 49, Centre de Mathématiques Appliquées, École Polytechnique, Palaiseau, 1979.

20. J. PASCIAK, "An iterative algorithm for the volume integral method for magnetostatics problems," Comput. Math. Appl., v. 8, 1982, pp. 283-290.

21. J. PASCIAK, "A new scalar potential formulation of the magnetostatic field problem," Math. Comp., v. 43, 1984, pp. 433-445.

22. I. I. PEKKER, "Calculation of magnetic systems by integration over field sources," Izv. Vyssh. Uchebn. Zaved. Electromekh., v. 9, 1964, pp. 1047-1051 (Russian)

23. W. Rudin, Principles of Mathematical Analysis, McGraw-Hill, New York, 1976.

24. J. Simkin \& C. W. Trowbridge, "On the use of total scalar potential in the numerical solution of field problems in electromagnetics,” Internat. J. Numer. Methods Engrg., v. 14, 1979, pp. 423-440.

25. J. Simkin \& C. W. Trowbridge, Three-Dimensional Computer Program (TOSCA) for Nonlinear Electromagnetic Fields, Rutherford Laboratory Report No. RL-79-097.

26. R. Temam, On the Theory and Numerical Analysis of the Navier Stokes Equations, North-Holland, Amsterdam, 1977.

27. M. M. VAInserg, Variational Method and Method of Monotone Operators in the Theory of Nonlinear Equations, Halsted Press, New York-Toronto, 1973. 